\title{
Relativistic electron fluxes in May 1992 and their effect on the middle atmosphere
}

\author{
E. E. Gaines, D. L. Chenette, and W. L. Imhof \\ Lockheed Palo Alto Research Laboratory, Palo Alto, California \\ C. H. Jackman \\ NASA Goddard Space Flight Center, Greenbelt, Maryland
}

\section{J. D. Winningham}

Southwest Research Institute, San Antonio, Texas

\begin{abstract}
Enhancements in the fluxes of relativistic electrons trapped within the Earth's magnetosphere have been measured by the high-energy particle spectrometer, part of the particle environment monitor on the upper atmosphere research satellite (UARS). The largest increase in the electron fluxes with energies greater than $1 \mathrm{MeV}$ observed on UARS from October 1991 through July 1994 was in early May 1992. The fluxes of trapped electrons in the drift loss cone and locally precipitating electrons showed differing buildup and decay rates as a function of invariant latitude. Increases of more than 2 orders of magnitude were observed in drift loss cone fluxes at magnetic latitudes of $40^{\circ}-66^{\circ}$ and in precipitating fluxes from $48^{\circ}$ to $66^{\circ}$. The energy flux contained in the most intense local precipitation observed was $\sim 0.1 \mathrm{erg} \mathrm{cm}^{-2} \mathrm{~s}^{-1}$, entering the atmosphere and creating up to 1000 ion pairs $\mathrm{cm}^{-3} \mathrm{~s}^{-1}$ at $55-\mathrm{km}$ altitude. The daily averaged energy flux from directly precipitating electrons with energies $>1$ $\mathrm{MeV}$ deposited $>10^{20} \mathrm{erg} \mathrm{d}^{-1}$ worldwide into the atmosphere for the period May 1221,1992 , producing $>10^{31}$ odd nitrogen molecules below $60-\mathrm{km}$ altitude.
\end{abstract}

\section{Introduction}

Enhancements in relativistic electron fluxes continue to command interest in the magnetospheric physics community and may be an important source of energy input and chemical change to the middle atmosphere [Thorne, 1980; Baker et al., 1987, 1990; Callis et al., 1991; Jackman, 1991]. These electrons are believed to be accelerated as a result of high-speed solar wind streams interacting with the magnetosphere [Paulikas and Blake, 1979; Baker et al., 1994] and may appear more frequently near solar minimum than solar maximum [Baker et al., 1986].

Since electrons with energies $>1 \mathrm{MeV}$ can penetrate to altitudes of $50 \mathrm{~km}$ and below, they can affect atmospheric chemistry and dynamics throughout the mesosphere. One of the objectives of the particle environment monitor (PEM) investigation on the upper atmosphere research satellite (UARS) is to measure these precipitating particles, including how they are distributed in energy, space, and time, and to use these measurements to assess the relative importance of the effects of their energy deposition on the middle atmosphere.

Callis et al. [1991] have concluded that relativistic electron precipitation is a significant source of odd nitrogen in the middle atmosphere. They also suggested that this source should contribute significantly to large losses of ozone observed in some regions of the atmosphere. Their analysis

Copyright 1995 by the American Geophysical Union.

Paper number 94JD02615.

0148-0227/95/94JD-02615\$05.00 was based on electron flux measurements made at geosynchronous orbit.

Estimating the flux precipitating into the atmosphere from geosynchronous measurements is subject to significant uncertainties because the measurements do not cover the peak of the trapped electron flux radial distribution. They map to a limited region in latitude at the top of the atmosphere, and only a small fraction of the flux measured at the high-altitude equator can reach low altitudes to penetrate into the atmosphere. From a low-altitude satellite like UARS, however, in its $57^{\circ}$ inclination orbit, global-scale coverage is obtained daily, and the fraction of the particle flux precipitating into the atmosphere can be measured directly with instruments of moderate angular resolution such as the high-energy particle spectrometer (HEPS) in PEM.

In this paper we summarize the relativistic electron precipitation during the period May 8-27, 1992. This period included the largest relativistic electron enhancement observed by PEM from the launch of UARS in September 1991 [Gaines et al., 1993] through July 1994. The spatial and temporal behavior of the measured precipitating electrons and their distribution in energy are presented. These results are used to calculate the altitude profile of energy deposition and ionization in the atmosphere and from this to estimate the global rate of odd nitrogen production in the mesosphere.

\section{Instrumentation}

HEPS is part of the PEM on the UARS, a three-axis stabilized vehicle in a $585 \mathrm{~km}, 57^{\circ}$ inclination orbit. HEPS provides high-sensitivity measurements of protons and elec- 
trons over a wide range of energies with good resolution and pitch angle sampling. The instrument includes four solidstate detector telescopes with geometric factors of $0.54 \mathrm{~cm}^{2}$ sr pointed at angles of $-15^{\circ},+15^{\circ},+45^{\circ}$, and $+90^{\circ}$ from the zenith, along with two low-energy proton detectors at $-15^{\circ}$ and $+15^{\circ}$ to the zenith. The energy ranges measured are $30 \mathrm{keV}$ to $5 \mathrm{MeV}$ for electrons and $60 \mathrm{keV}$ to $150 \mathrm{MeV}$ for protons. A more complete description of HEPS and the other PEM instruments is given by Winningham et al. [1993].

As the spacecraft travels in orbit, the angles between the geomagnetic field vector and the collimator axes of the telescopes (pitch angles for particles) change, necessitating computation of the local pitch angle for all sensors at each data point. This has been incorporated into the data reduction software.

\section{Data Presentation}

For this analysis, energetic electron measurements are divided into three groups depending on pitch angle and location. Bounce loss cone (BLC) electrons are in the angular range from downward along the direction of the magnetic field out to the pitch angle corresponding to a mirror altitude of $100 \mathrm{~km}$. They are "locally precipitating" and considered to be lost to the atmosphere immediately. All other electrons are "locally trapped." Drift loss cone (DLC) electrons [Luhmann and Vampola, 1977] are those locally trapped electrons which will be in the BLC at some point in their azimuthal drift around the Earth (due to azimuthal asymmetries in the magnetic field only and assuming no additional pitch angle scattering). The remaining electrons are considered to be stably trapped for this study; that is, they are not expected to enter the atmosphere on a timescale comparable to those for the BLC $(<1 \mathrm{~s})$ and DLC ( $<1$ hour) fluxes.

Whole days of HEPS observations were sorted into locally trapped and locally precipitating fluxes as a function of magnetic $L$ shell in $\Delta L=0.5$ bins. Whole days were used to help ensure even sampling at all longitudes. Locally precipitating fluxes were so labeled only if the field of view of a sensor was completely within the BLC. This results in an underestimation of the total local precipitating flux since the true detection efficiency falls off toward the edge of the field of view, and the precipitating flux is typically more intense closer to the edge of the loss cone [Imhof and Gaines, 1993]. The magnitude of this effect is, of course, dependent on the shape of the pitch angle distribution within the loss cone. Calculations using HEPS collimator angular responses with pitch angle distributions similar to those measured with fine angular resolution [Imhof and Gaines, 1993] that fit several HEPS measured distributions ( $L=3-4)$ showed factors of 2-5 more flux at the edge of the BLC than HEPS would measure.

At the altitude of UARS, locally trapped electrons are all in the DLC except in a region over South America and the South Atlantic Ocean, and trapped flux data from this region have been excluded from the present analysis.

The geographic patterns of these fluxes are illustrated in Plate 1 for May 12, 1992 and are similar for other days during the event. The intense fluxes of trapped electrons between South America and Africa extending to $60^{\circ} \mathrm{E}$ longitude are stably trapped. This is the South Atlantic Anomaly, the region of lowest magnetic field intensity. DLC fluxes in both hemispheres can be seen to follow lines of constant geomagnetic latitude or equivalently constant $L$ shells as shown by dotted lines. The BLC precipitating fluxes generally exhibit a similar pattern but with intensities lower by about an order of magnitude than the trapped fluxes except for the $L$ band 2.0-2.5 as will be discussed later in the text. Note that the maximum BLC flux coincides with the stably trapped flux maximum.

The time behavior of the fluxes of electrons with energies greater than $1 \mathrm{MeV}$ at various invariant latitudes or magnetic $L$ shells during the enhancement event in May 1992 will be examined first. DLC fluxes are shown in Figure 1 with the geomagnetic indices $\mathrm{K}_{\mathrm{p}}$ and $\mathrm{D}_{\mathrm{st}}$ for reference. For $L$ values above 4.5, electrons from the solar particle event are evident on May 9 with a sharp decay to May 10 before the buildup begins. In Figure 2 the BLC fluxes are presented on the same timescale. Temporal behavior similar to that in the DLC trapped fluxes appears; however, the enhancement in the precipitating fluxes does not extend as low in $L$ value as do the DLC trapped fluxes, nor are the decay and a second enhancement in these fluxes evident in the BLC electron population. In fact, the BLC flux in the $L$ band 3.0-3.5 does not appear to decay at all between May 12 and 21. These temporal patterns are not similar to those in the superposed epoch analysis of several smaller events presented by Baker et al. [1994] and may indicate that this event period starting with a very large magnetic disturbance is atypical. At the times of peak flux intensity in the DLC, these fluxes are greater than the BLC fluxes by factors of 10-100.

Figure 3 shows energy spectra of DLC and locally precipitating electrons from selected times for $L=3.5-4.0$ during the enhancement and from a geomagnetically quiet day, April 14, 1992, when the relativistic electron fluxes were not significantly enhanced. The peak in the precipitating fluxes (2245-2249 UT) on May 18 heavily weights the average spectrum for that day. This spectrum is much harder than for the May 14 day average, and the fluxes are 3 orders of magnitude greater than the day-averaged fluxes on April 14 at energies greater than $2 \mathrm{MeV}$. The May 14 spectrum is more typical of the BLC fluxes for the overall period. There is less variation in spectral shape for the DLC fluxes during the enhanced period, and the examples from May 14 and 18 are representative for the period.

The ion production profiles in Figure 4 were obtained from the spectra in Figure 3 using the method developed by $C$. $H$. Jackman [see Goldberg et al., 1984]. The solid line curves are for directly precipitating electrons, and the dashed line is for the May $14 \mathrm{DLC}$ electrons at $80^{\circ} \mathrm{W}$ longitude (assuming that this flux does not experience pitch angle scattering in its further drift and is isotropic over the downward direction at its precipitation point). Spectra for these calculations included the entire energy range ( $30 \mathrm{keV}$ to $5 \mathrm{MeV}$ ), the higher energy portion of which is shown in Figure 3. The softest spectrum (April 14, 1992) results in ionization exceeding that of cosmic rays at altitudes above $\sim 53 \mathrm{~km}$ and peaking at 69 $\mathrm{km}$. The second peak near $30 \mathrm{~km}$ in all of the curves is from absorption of bremsstrahlung produced by the electrons that stop higher in the atmosphere. Ionization from the May 14, 1992, day-averaged spectrum is more than an order of magnitude more intense than on April 14, exceeding the cosmic ray rate above $43 \mathrm{~km}$. Both exhibit a maximum rate near $65 \mathrm{~km}$. The ionization profile from the May 14 localized 

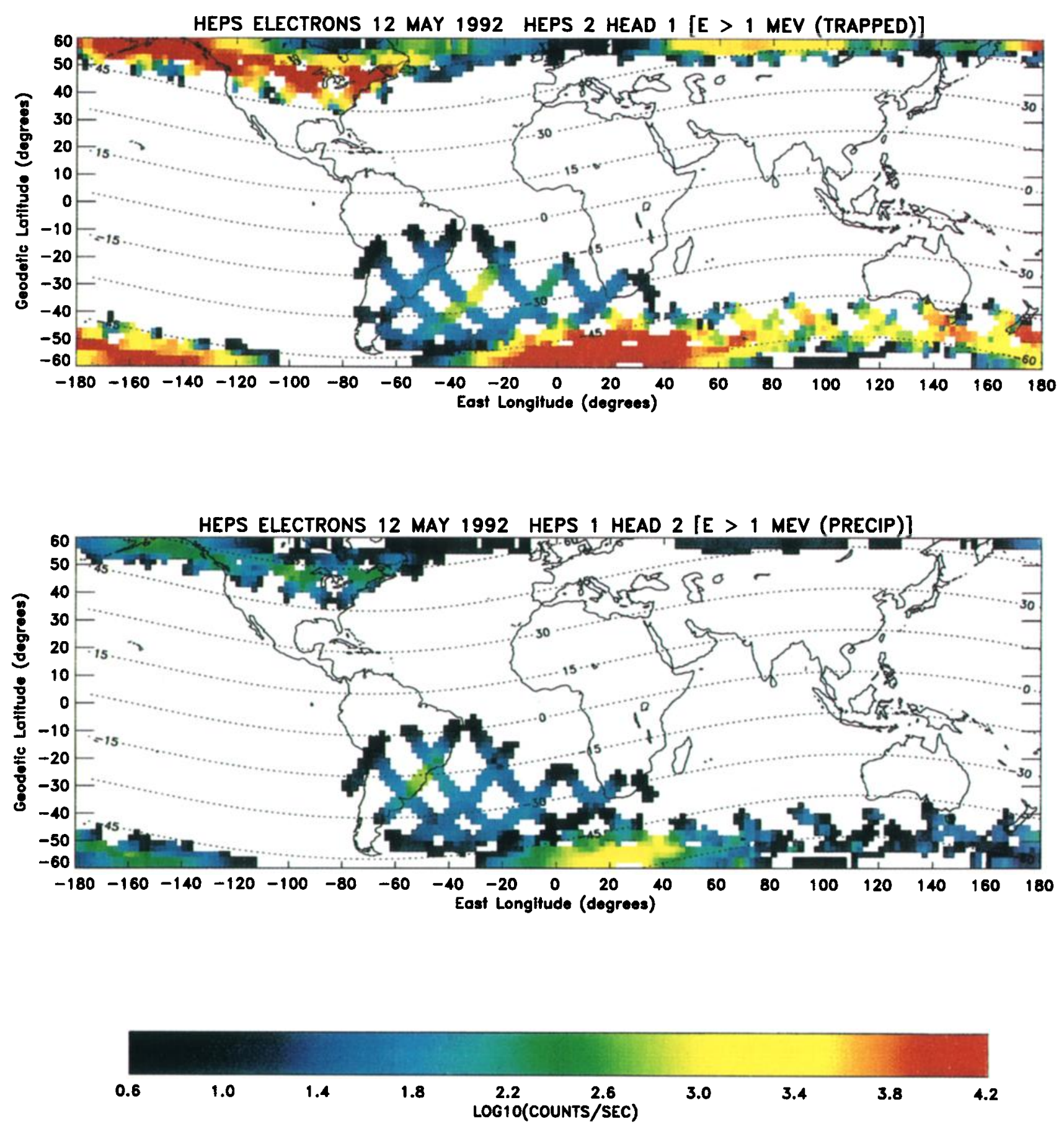

Plate 1. The geographic distribution of locally trapped and precipitating electrons (bounce loss cone) with energies greater than $1 \mathrm{MeV}$ measured from UARS on May 12, 1992. Lines of constant geomagnetic latitude are indicated by dotted lines.

DLC flux is greater than that from the peak of the direct precipitation above about $57-\mathrm{km}$ altitude. The harder spectrum from the BLC electrons produces the increased ionization down to $30 \mathrm{~km}$. The May 14 DLC and May 18 peak BLC profiles are 2-3 orders of magnitude greater than on April 14 and dominate cosmic ray rates down to $\sim 30 \mathrm{~km}$.

In Figure 5 the integrated energy from both populations of electrons for selected day-averaged spectra is plotted versus magnetic invariant latitude. From these, estimations of the global energy input to the atmosphere can be made.

\section{Discussion}

Electrons in the DLC may be the major source of energy above $40 \mathrm{~km}$ from charged particles precipitating into the atmosphere at certain times and locations. The contribution from these fluxes precipitating in a restricted longitude region relative to that from the more widely distributed BLC fluxes during this enhancement period is therefore important to ascertain.

The enhanced fluxes of DLC electrons $>1 \mathrm{MeV}$ beginning 


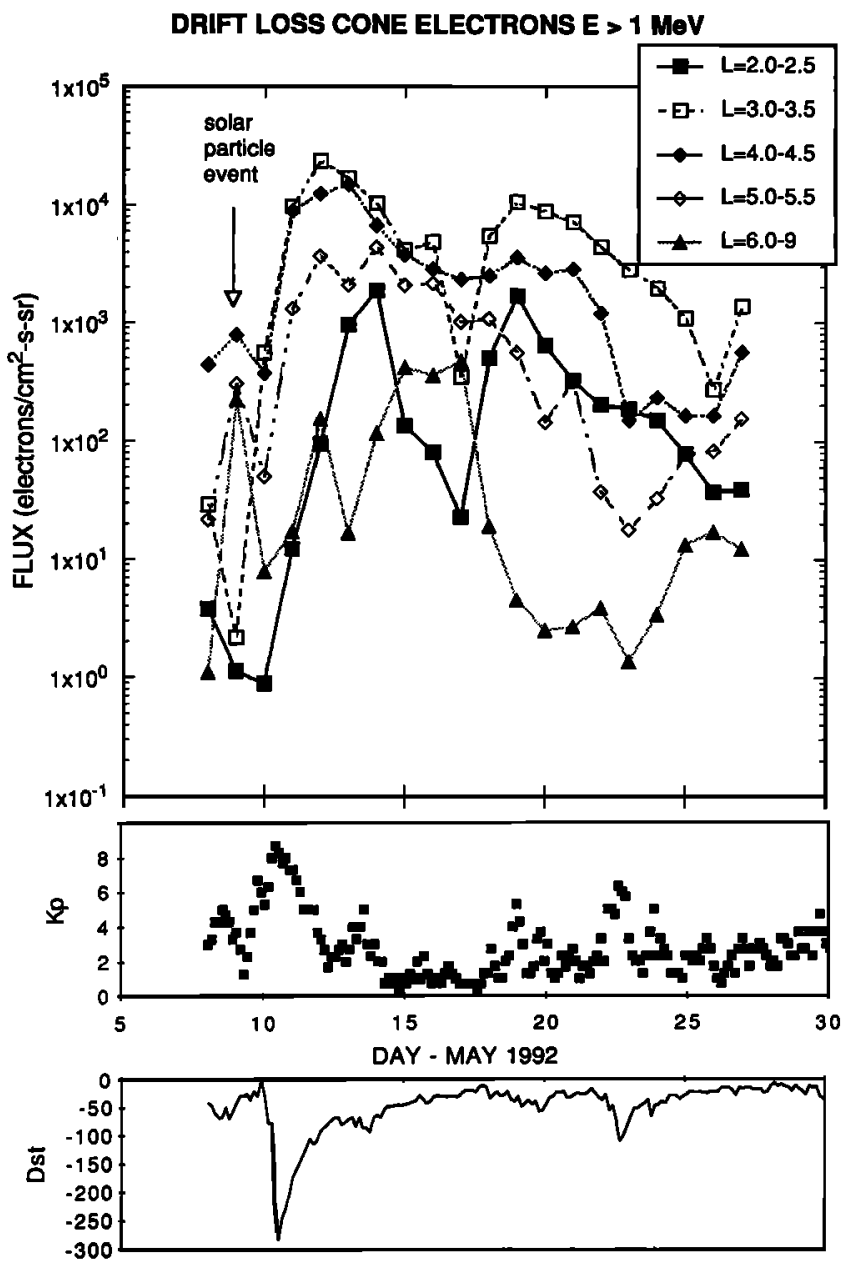

Figure 1. Drift loss cone trapped electron fluxes with energies greater than $1 \mathrm{MeV}$ versus time in May 1992 for selected magnetic $L$ shell intervals along with the geomagnetic indices $K p$ and $D s t$.

May 10, 1992, and presented in Figure 1 show similar time profiles for $L=2.5-4.0$ with an initial peak on May 12-13, a rapid decay to May 17, and a second buildup peaking on May 19. Fluxes at $L=2.0-2.5$ show the same initial rapid rise but delayed by about 1 day. In the higher $L$ regions $(4.0-6.0)$ the fluxes do not drop sharply on May 17 but show differing decay rates thereafter. For $L>6$ the flux behavior is much more dynamic with changes of an order of magnitude in a day. For all but the region $L=4.0-4.5$, the maximum flux enhancements are more than 2 orders of magnitude above the preevent values.

The BLC fluxes for $L>4$ exhibit patterns that are similar to each other. For $L=3.0-4.0$, after the initial buildup to May 12, the fluxes are remarkably steady until May 21, at which time a sharp decrease of a factor of 5 occurs. The fluxes at $L<2.5$ show little variation in contrast to those in the DLC. These temporal patterns are not similar to those in the superposed epoch analysis of several smaller events presented by Baker et al. [1994] and may indicate that this event period starting with a very large magnetic disturbance is atypical.

Overall, the BLC fluxes that encompassed the most global area $(L=2.5-4.0)$ and contributed most to the global atmospheric energy input were fairly constant between May
12 and 21,1992 . The geographic distribution of the BLC fluxes is not uniform in longitude, however. The peak precipitation intensities occur in the region with the maximum fluxes of stably trapped electrons south of Africa.

The UARS $57^{\circ}$ orbital inclination precludes measurement of some of the electrons in the highest flux interval $(L=$ -3-4). In each hemisphere the excluded longitudes are about one half of the total. On the basis of previous observations of the electron flux dependence on magnetic field intensity and model field strengths in the unsampled areas, fluxes north of $57^{\circ}$ latitude are not expected to be greater than the HEPS measured average. In the southern hemisphere, however, the fluxes south of $57^{\circ}$ would be expected to range from the maximum seen in Plate 1 near $0^{\circ}-20^{\circ} \mathrm{E}$ longitude to those around $160^{\circ} \mathrm{W}$ at the southern limit of the orbit. Quantitatively, the maximum BLC flux $>1 \mathrm{MeV}$ is a factor of 6-10 greater than the day average $L=3-4$ flux over the period May 12-21. Thus the average flux in the southern excluded area should be no greater than 3-5 times the daily average measured flux for $L=3-4$. Assuming that this flux fills $25 \%$ of the area of the $L$ band, the actual flux, had the coverage been complete, would be no more than a factor of 2 greater than the measured average flux.

Previous analysis of fine pitch angle resolution of the DLC fluxes compared with those in the BLC as a function of longitude at $600 \mathrm{~km}$ [Imhof and Gaines, 1993] suggests that over the range of the DLC measurements at $585 \mathrm{~km}$, much of this flux scatters into the BLC as it drifts eastward. The maximum DLC fluxes measured west of the stable trapping region are not sufficiently intense so that when integrated over the precipitation area, the total number of precipitated electrons is smaller than that for BLC electrons by more than an order of magnitude.

The first enhancement in fluxes is coincident with a very rapid rise in solar wind velocity measured from IMP 8 late on May 9, coinciding with the start of rapid increases in $K_{p}$ and

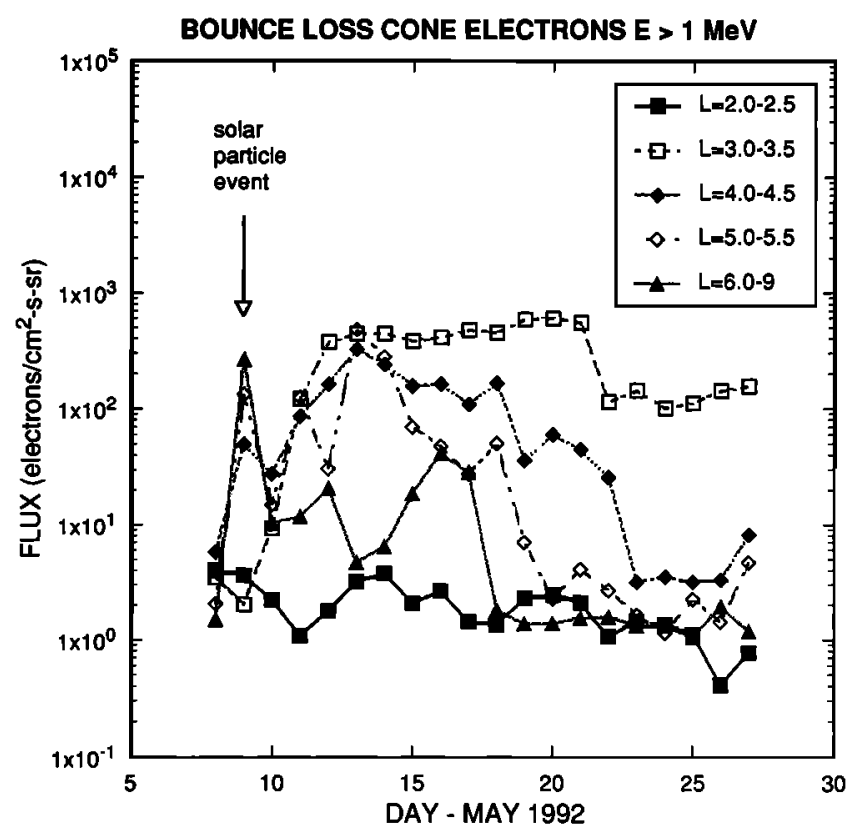

Figure 2. Daily averaged precipitating (bounce loss cone) electron fluxes with energies greater than $1 \mathrm{MeV}$ versus time in May 1992 for selected $L$ shell intervals. 

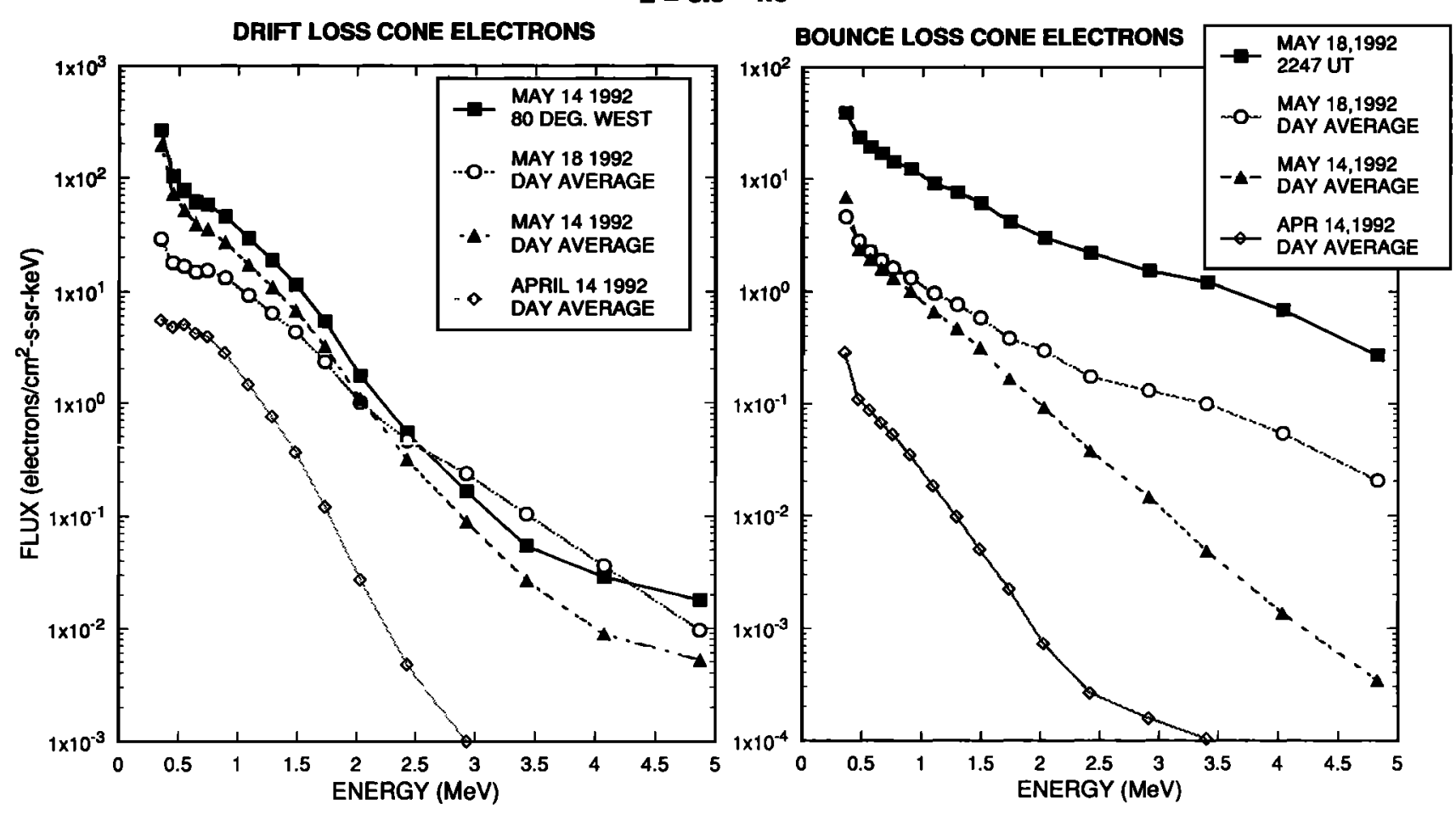

Figure 3. Energy spectra of precipitating and drift loss cone electrons in the $L$ interval $3.5-4.0$ for a magnetically quiet day, April 14, 1992, and for selected times during the enhancement in May 1992.

$D_{\text {st }}$ as shown in Plate 1. A lack of solar wind data from May 10-15 precludes this evaluation for the second increase; however, a small perturbation in the geomagnetic indices does occur beginning late on May 12 and might be a signature of another electron acceleration.

The energy spectra during the period of enhancement, examples of which are shown in Figure 3, can be reasonably well described analytically above $1 \mathrm{MeV}$ by exponentials with $e$-folding energies of $\sim 0.4-1 \mathrm{MeV}$.

The spectra for May 14, 1992, are typical of those for $L=$ 3.0-4.5 over the time period May 12-21. Thus the ionization profile shown in Figure 4 from the day average spectrum of precipitating electrons for May 14 is representative of the period. The ionization produced at altitudes below $60 \mathrm{~km}$ is

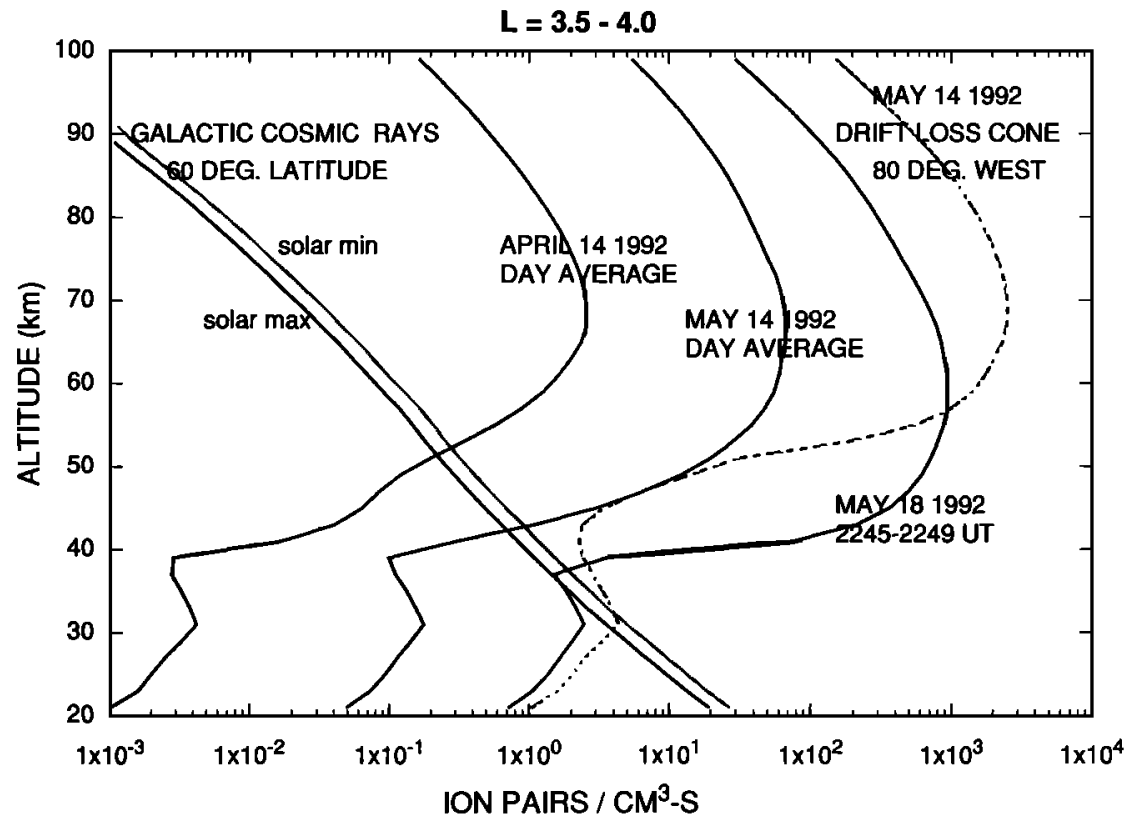

Figure 4. Ion production profiles from electron spectra in Figure 3 and those due to galactic cosmic rays at $60^{\circ}$ magnetic latitude. Solid curves are for directly precipitating electrons. 


\section{DRIFT LOSS CONE ELECTRONS E $>1 \mathrm{MeV}$}

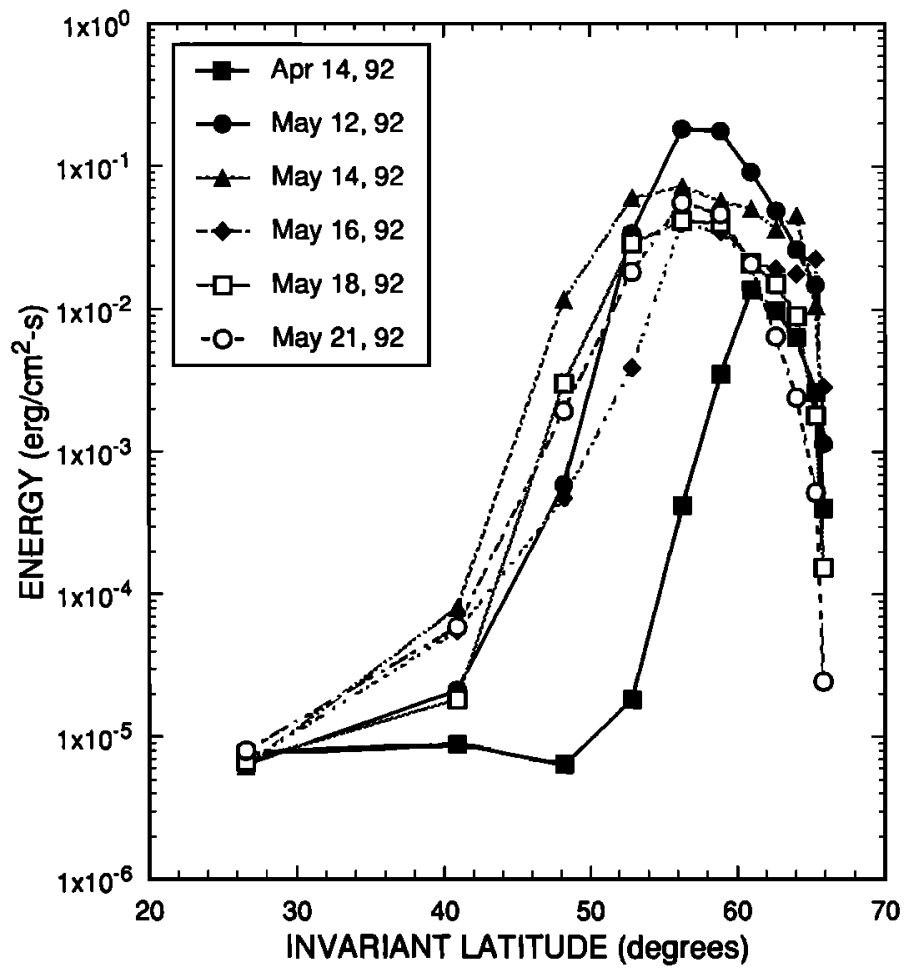

BOUNCE LOSS CONE ELECTRONS E > 1 MeV

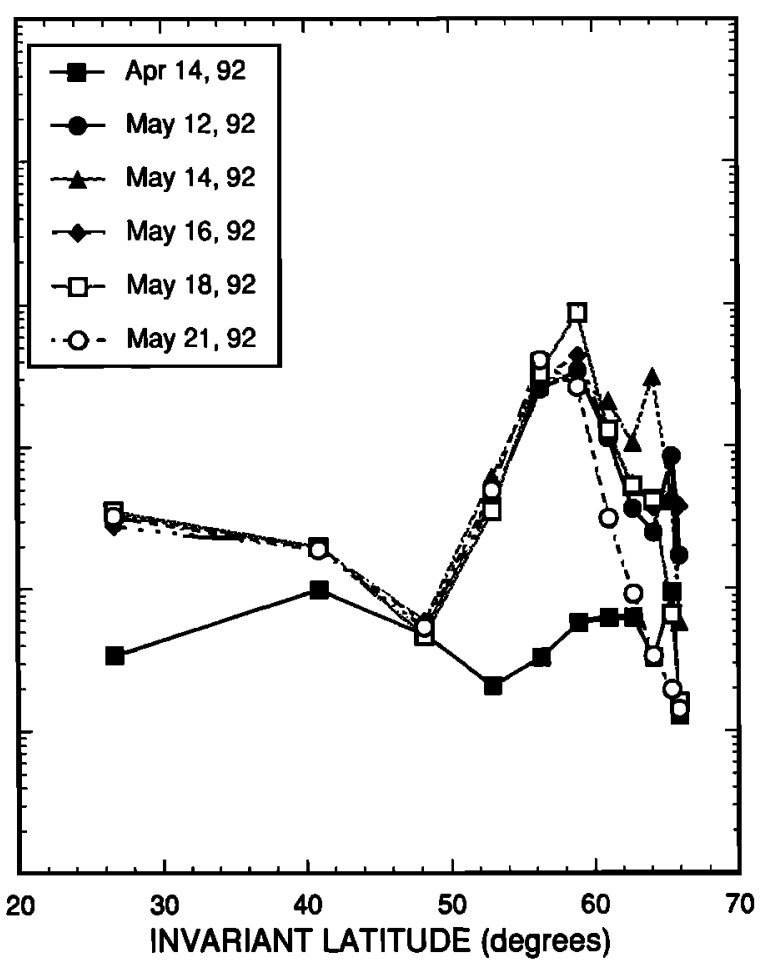

Figure 5. Energy deposition into the atmosphere from precipitating electrons in both the bounce and drift loss cones with energies greater than $1 \mathrm{MeV}$ versus magnetic invariant latitude on April 14, 1992, and selected days through the May 1992 enhancement event.

an order of magnitude or more greater than that from the magnetically quiet day, April 14. The highest intensity short-term electron precipitation observed on May 18 (22452249 UT) produced more than an order of magnitude greater ionization than the day average fluxes; however, this was over a much smaller area than the global average.

The total energy entering the atmosphere from directly precipitating electrons can be obtained from the BLC data in Figure 5, which shows that from May 12 to 21, the daily average energy input as a function of invariant latitude was rather constant up to $60^{\circ}(L=4)$ with the exception of the peak precipitation on May 18 . When the average energy from the directly precipitating electrons in each segment of invariant latitude is multiplied by the segment area, the resultant global energy input is $>10^{20} \mathrm{erg} \mathrm{d}^{-1}$ for the entire period between May 12 and 21. This is a lower limit based only on the fluxes actually measured at UARS and a conservative definition of the fluxes in the BLC. The factors for the flux not measured near the edge of the loss cone and for orbital coverage exclusion could increase this global energy value by a factor of $4-10$. The inclusion of electrons between 0.3 and $1 \mathrm{MeV}$ in the calculated energy deposition results in a factor of 2.6 greater energy than that from $E>1 \mathrm{MeV}$ electrons. Most of this energy would be deposited between 60 and $66 \mathrm{~km}$. Energy deposition of this magnitude at altitudes below $\sim 60 \mathrm{~km}$ can significantly affect atmospheric chemistry. Production of shorter-lived species such as hydrogen radicals $\left(\mathrm{HO}_{\mathrm{x}}\right)$ may be important because of the 10-day duration and geographical distribution to lower latitudes of the electron precipitation. These products are important in mesospheric ozone destruction. For the longerlived $\mathrm{NO}_{\mathrm{y}}$ the total production in the lower mesosphere over the time period is important because it can be transported to lower altitudes and latitudes. This total $\mathrm{NO}_{\mathrm{y}}$ production resulting from the lower limit measurement of the BLC electron fluxes for the enhancement period is estimated to be $\sim 10^{31}$ molecules created between $55^{\circ}$ and $60^{\circ}$ invariant latitude and from 40 to $60 \mathrm{~km}$ in altitude with a possible enhancement of as much as a factor of 2 from transport of $\mathrm{NO}_{\mathrm{y}}$ created above $60 \mathrm{~km}$. This lower limit value is $\sim 0.5-$ $1.0 \%$ of the global annual source from $\mathrm{N}_{2} \mathrm{O}$ oxidation at latitudes greater than $50^{\circ}$ calculated by Jackman et al. [1990] and $\sim 0.1-0.2 \%$ of the ambient global $\mathrm{NO}_{\mathrm{y}}$ content calculated by Callis et al. [1991]. The true total production value could be an order of magnitude greater if incomplete orbital and instrumental pitch angle sampling factors are included. While this electron source of odd nitrogen is small compared to the global values, it can be dominant in localized regions during the periods of precipitation and may be detectable in other instruments on UARS. In order to understand the longer-term contribution of relativistic electrons to the $\mathrm{NO}_{\mathrm{y}}$ production in the middle atmosphere, electron events over longer time periods (such as years or solar cycles) should be quantified in a way similar to that used in this analysis.

\section{Conclusions}

1. The relativistic electron flux enhancement of May 1992 is the most intense measured from UARS to date, and the increased fluxes of $>1 \mathrm{MeV}$ electrons extended deeper 
into the magnetosphere (to lower magnetic latitudes) than previously reported events.

2. The rate of energy deposition into the atmosphere from these electron fluxes was 2 orders of magnitude or more greater than during a magnetically undisturbed time at altitudes below $60 \mathrm{~km}$ in the invariant latitude region $\sim 55^{\circ}-60^{\circ}$.

3. The energy deposited into the atmosphere by precipitating electrons $>1 \mathrm{MeV}$ observed in the BLC is greater than that from DLC electrons by more than an order of magnitude except for $L=2.0-2.5$, the innermost extent of the flux enhancement.

4. The geographic location of the most intense measured electron precipitation is centered at about $20^{\circ} \mathrm{E}$ longitude and southward of $50^{\circ} \mathrm{S}$ latitude coincident with maximum fluxes of stably trapped electrons. The effects of this peak energy deposition on the chemistry of the lower mesosphere may be detectable from UARS.

5. Ionization in the altitude range $40-60 \mathrm{~km}$ from $\mathrm{BLC}$ electrons $>1 \mathrm{MeV}$ is estimated to have produced $>10^{31}$ molecules of odd nitrogen during the flux enhancement period, small fractions of the previously estimated annual source and ambient global content that may nonetheless be significant because of the relatively short production time.

\section{Future Work}

Correlation of measured electron precipitation with $\mathrm{NO}_{2}$ measurements from the UARS Cryogenic Limb Array Etalon Spectrometer (CLAES) during the event will be undertaken, although its view direction was not optimum at the time. Also, comparisons of other events with that of May 1992 will be of interest to show the relative importance of this one large electron flux enhancement to the frequent smaller ones observed since that time as we approach solar minimum.

Acknowledgments. IMP 8 data used in this study were provided by WDC-A for Solar-Terrestrial Physics, NOAA E/GC2, 325 Broadway, Boulder, CO 80383.

\section{References}

Baker, D. N., J. B. Blake, R. W. Klebesadel, and P. R. Higbie, Highly relativistic electrons in the Earth's outer magnetosphere, 1, Lifetimes and temporal history 1979-1984, J. Geophys. Res., $91,4265,1986$

Baker, D. N., J. B. Blake, D. J. Gorney, P. R. Hughes, R. W. Klebesadel, and J. H. King, Highly relativistic magnetospheric electrons: A role in coupling to the middle atmosphere?, Geophys. Res. Lett., 14, 1027, 1987.

Baker, D. N., J. B. Blake, R. W. Klebesadel, D. D. Sentman, D. J. Gorney, and P. R. Higbie, Relativistic magnetospheric electrons: Lower ionospheric conductivity and long-term atmospheric variability, Adv. Space Res., 10, 229, 1990.

Baker, D. N., J. B. Blake, L. B. Callis, J. R. Cummings, D. Hovestadt, S. Kanekal, B. Klecker, R. A. Mewald, and R. D. Zwickl, Relativistic electron acceleration and decay timescales in the inner and outer radiation belts: SAMPEX, Geophys. Res. Lett., 21, 409, 1994.

Callis, L. B., D. N. Baker, J. B. Blake, J. D. Lambeth, R. E. Boughner, M. Natarajan, R. W. Klebesadel, and D. J. Gorney, Precipitating relativistic electrons: Their long-term effect on stratospheric odd nitrogen, J. Geophys. Res., 96, 2939, 1991.

Gaines, E. E., D. L. Chenette, R. W. Nightingale, W. L. Imhof, and J. D. Winningham, Relativistic electron flux enhancements observed by the particle environment monitor on UARS, Eos Trans. $A G U, 74(43)$, Fall Meeting suppl., 517, 1993.

Goldberg, R. A., C. H. Jackman, J. R. Barcus, and F. Soraas, Nighttime auroral energy deposition in the middle atmosphere, $J$. Geophys. Res., 89, 5581, 1984.

Imhof, W. L., and E. E. Gaines, Inputs to the atmosphere from relativistic electrons, J. Geophys. Res., 98, 13,575, 1993.

Jackman, C. H., Effects of energetic particles on minor constituents of the middle atmosphere, J. Geomagn. Geoelectr., 43, suppl., 637, 1991.

Jackman, C. H., A. R. Douglass, R. B. Rood, and R. D. McPeters, Effect of solar proton events on the middle atmosphere during the past two solar cycles as computed using a two-dimensional model, J. Geophys. Res., 95, 7417, 1990.

Luhmann, J. G., and A. L. Vampola, Effects of localized sources on quiet time plasmasphere electron precipitation, J. Geophys. Res., 82, 2671, 1977.

Paulikas, G. A., and J. B. Blake, Effects of the solar wind on magnetospheric dynamics: Energetic electrons at the synchronous orbit, in Quantitative Modeling of Magnetospheric Processes, Geophys. Monogr. Ser., vol. 21, edited by W. B. Olson, pp. 180-202, AGU, Washington, DC, 1979.

Thorne, R. M., The importance of energetic particle precipitation on the chemical composition of the middle atmosphere, Pure Appl. Geophys., I18, 128, 1980.

Winningham, J. D., et al., The UARS particle environment monitor, J. Geophys. Res., 98, 10,649, 1993.

D. L. Chenette, E. E. Gaines, and W. L. Imhof, Lockheed Palo Alto Research Laboratory, Department 91-20, Bldg. 252, 3251

Hanover Street, Palo Alto, CA 94304-1191. (e-mail: lockhd::gaines)

C. H. Jackman, NASA Goddard Space Flight Center, MC916, Greenbelt, MD 20771.

J. D. Winningham, Southwest Research Institute, P. O. Drawer 28510, San Antonio, TX 78284.

(Received April 28, 1994; revised September 25, 1994; accepted September 25, 1994.) 